Research Paper: Autophagy and Cell Death

\title{
Aberrant localization of apoptosis protease activating factor-1 in lipid raft sub-domains of diffuse large B cell lymphomas
}

\author{
Jayshree L. Hirpara ${ }^{1,2}$, Thomas Loh ${ }^{3}$, Siok Bian $\mathrm{Ng}^{4}$, Wee Joo $\mathrm{Chng}^{5}$ and Shazib \\ Pervaiz ${ }^{1,6,7,8}$ \\ ${ }^{1}$ Department of Physiology, Yong Loo Lin School of Medicine, National University of Singapore, Singapore \\ 2 Experimental Therapeutics Program, Cancer Science Institute, National University Healthcare System, Singapore \\ ${ }^{3}$ Department of Otolaryngology, National University Healthcare System, Singapore \\ ${ }^{4}$ Department of Pathology, National University Healthcare System, Singapore \\ ${ }^{5}$ Cancer Science Institute, National University Healthcare System, Singapore \\ ${ }^{6}$ NUS Graduate School for Integrative Sciences and Engineering, National University of Singapore, Singapore \\ 7 National University Cancer Institute, National University Healthcare System, \\ ${ }^{8}$ School of Biomedical Sciences, Curtin University, Perth, Australia \\ Correspondence to: Shazib Pervaiz, email: Shazib_Pervaiz@nuhs.edu.sg
}

Keywords: DLBCL, apoptosome, Apaf-1, lipid rafts, ROS, Autophagy

Received: April 15, $2016 \quad$ Accepted: November 08, $2016 \quad$ Published: November 14, 2016

\section{ABSTRACT}

Resistance to chemotherapy remains a challenge in the clinical management of diffuse B cell lymphomas despite aggressive chemotherapy such as CHOP and monoclonal CD20. Here we provide evidence that the apoptosome adaptor protein, Apaf-1, is mislocalized in primary cells derived from patients with diffuse large $B$ cell lymphomas (DLBCL). Whereas, the total expression of Apaf-1 did not change, its subcellular localization was significantly different in DLBCL, compared to $T$ cell lymphomas as well as cells derived from reactive lymphadenopathy biopsies. As expected, Apaf-1 was detected in the cytosolic fractions of non-B cell lymphomas and non-cancerous tissues; however, in B cell derived lymphomas the protein was detected in membrane raft sub-domains rather than the cytosol. Disruption of lipid raft structures resulted in the redistribution of Apaf-1 to the cytosol and restored apoptosis sensitivity of DLBCL. Furthermore, we identified novel small molecule compounds that target DLBCL by promoting Apaf-1 release form lipid rafts via mechanisms that involve an increase in intracellular reactive oxygen species production. Taken together, our results implicate Apaf-1 mislocalization as a potential diagnostic and prognostic marker for DLBCL, and provide a novel therapeutic strategy for circumventing the drug refractory nature of this sub-class of B cell lymphoma.

\section{INTRODUCTION}

Non-Hodgkin Lymphoma (NHL) is a diverse group of cancers affecting the lymphatic/hematopoietic system. There are at least 30 types of NHL, with diffuse large B-cell lymphoma (DLBCL) and Follicular lymphoma being the most common and aggressive types of NHL. With timely and appropriate chemotherapy, such as CHOP and monoclonal CD20 (bivatuzamab), over half of the DLBCL patients are cured [1]; however, chemoresistance remains a major challenge, which potentiates the need for an improved chemotherapeutic strategy in relapsed patients. The molecular mechanisms underlying the resistance of DLBCL to chemotherapy include overexpression of the anti-apoptotic proteins belonging to the Bcl-2 family and/or the IAP (inhibitor of apoptosis proteins) family, thereby resulting in dysregulation of the apoptotic execution machinery [1].

The apoptotic program is orchestrated by a cascade of events resulting in the activation of caspases, either through the ligation of the death receptor (extrinsic signaling) or by engaging the mitochondrial amplification pathway (intrinsic signaling). The intrinsic death pathway is the common mode of chemotherapy- 
induced execution and requires the permeabilization of the outer mitochondrial membrane and release of death amplification proteins such as cytochrome C, Smac and AIF [2]. The cytosolic translocation of cytochrome $\mathrm{C}$ is critical for the formation and activation of the apoptosome, a death-signaling complex downstream of the mitochondria $[3,4]$. The assembly of the apoptosome is brought about by the recruitment of cytochrome $\mathrm{C}$ and pro-caspase 9 to the adaptor protein Apaf-1 (Apoptosis Protease Activating Factor 1) in the presence of dATP, resulting in the activation of caspase 9, which then drives the activation of the executioner caspase $3[5,6]$. Hence, death inhibitory proteins such as Bcl-2 indirectly inhibit the assembly of the apoptosome by regulating mitochondrial outer membrane permeabilization, while others such as the IAPs (Inhibitor of Apoptosis Proteins) control apoptotic execution downstream of the apoptosome by targeting caspase 9 [3].

There is ample evidence to implicate defective apoptosome assembly in the resistance of cancer cells to drug-induced apoptosis [7]. To that end, a number of recent reports demonstrate a link between chemoresistance and the cellular levels of Apaf-1. For example, epigenetic regulation of Apaf-1 via its promoter methylation is associated with apoptosis resistance in malignant melanoma cells [8] as well as in some leukemias [9, 10]; however, in another study, inactivation of Apaf-1 was not corroborated in malignant melanoma [11] Defective apoptosome formation through $\mathrm{LOH}$-mediated repression of Apaf-1 has also been reported in glioblastoma [10] and Apaf-1 hypermethylation has been seen in bladder cancer [12]. Furthermore, loss of Apaf-1 has been linked to tumor aggressiveness in cervical cancer [13]. Corroborating the association between an absence or downregulation of Apaf-1 expression and drug resistance and/or aggressiveness of cancer, we previously reported a hitherto undefined mechanism of defective apoptosome signaling in human B cell lymphoma cell lines by demonstrating the sequestration of Apaf-1 to the plasma membrane [14].

Here we set out to investigate the clinical relevance of Apaf-1 mislocalization in primary cells derived from patients with lymphomas, and to establish a correlation between Apaf-1 mislocalization and apoptosis sensitivity in an ex vivo setting. We report that Apaf-1 mislocalization to lipid raft fractions of the plasma membrane is associated with a significantly muted response to apoptosis stimuli in DLBCL and follicular lymphomas. Furthermore, we identified novel small molecules that restored chemosensitivity of B cell lymphomas by affecting the release of Apaf-1 to the cytosol through an increase in intracellular reactive oxygen species (ROS), thereby facilitating apoptosis execution. These data provide a novel mechanism of loss of apoptosome assembly and function and its association with apoptosis resistance in clinical B cell lymphomas, which could have potential implications for the design and development of novel therapeutic strategies against the aggressive and refractory variants of B cell lymphoma.

\section{RESULTS}

\section{Primary cells from B cell lymphoma patients are resistant to conventional chemotherapeutic agents}

In order to understand the precise mechanism underlying the resistance of human B cell lymphomas to drug-induced apoptosis, we obtained biopsies from patients with a variety of lymphomas and performed magnetic separation of $\mathrm{T}$ and $\mathrm{B}$ cells as described in Materials and Methods. Primary cells were then subjected ex vivo to apoptotic stimuli, including the commonly used chemotherapeutic agents etoposide $(1-10 \mu \mathrm{M})$, daunorubicin $(0.2-0.8 \mu \mathrm{g} / \mathrm{ml})$, vincristine $(1-10 \mu \mathrm{M})$, the death receptor ligand TRAIL $(50-200 \mathrm{ng} / \mathrm{ml})$, as well as experimental small molecule compounds LY30 (25$50 \mu \mathrm{M})[15], \mathrm{C} 1(25-100 \mu \mathrm{g} / \mathrm{ml})[16,17]$, MPO $(1-10 \mu \mathrm{M})$ [18], and MPO-Zn (100-400nM). As an internal control, two established cell lines, Raji and Jurkat, were used in parallel. Cell viability was assessed by the MTT assay following $24 \mathrm{~h}$ of incubation with the various compounds. Results show that primary cells from benign lymphomas or non-cancerous lymphoid hyperplasia were relatively insensitive to most drugs, while B cell lymphomas (BL Cells) in general were resistant to commonly used chemotherapeutic agents compared to $\mathrm{T}$ cell lymphomas (Figure 1a). This is in agreement with our earlier findings indicating B-cell lymphoma-derived cell lines were resistant to etoposide and other chemotherapeutic drugs. Interestingly, we provide evidence that primary BL cells were relatively more responsive to the experimental small molecules MPO[18], MPO-Zn (MPO analogue) and C1 $[16,17]$ (Figure 1b). We also tested the sensitivity of primary BL cells to the death receptor ligand TRAIL. TRAIL has been under clinical evaluation against because of its ability to selectively target cancer cells [19]. Firstly, primary BL cells, unlike TL cells, were not responsive to TRAIL, however pre-incubation of cells with the small molecule experimental compounds MPO, MPO-Zn, C1 or LY30 resulted in a significant amplification of TRAIL sensitivity (data not shown).

\section{Absence of Apaf-1 in the cytoplasm of primary BL cells}

The aforementioned results provided evidence that clinical B cell lymphomas in general were relatively refractory to known chemotherapeutic agents, but did possess the functional armory to elicit an apoptotic response depending on the nature of the stimulus. Intrigued by these findings and based on our earlier report 
using established cell line [14], we set out to investigate the role of the apoptosome machinery in the resistance of primary BL cells to drug-induced apoptosis. Primary cells derived from 30 B-cell lymphoma patients, 7 T-cell lymphoma patients and 5 benign tumors were used for the assessment of Apaf-1 expression and its localization. Interestingly, Apaf-1 expression was detected in the S100 cytosolic fractions from primary TL cells as well as Jurkat cell line, however, the expression was virtually completely absent in the S100 cytosol but found mostly in the S100 pellet fractions of primary BL cells (Figure 1c, Supplementary Figure1a, b and c).

\section{Disruption of Apaf-1 from S-100 pellet increases caspase-3 activity}

Apaf-1 is an essential adaptor protein required for the assembly of the apoptosome, which brings about the activation of downstream caspases, such as caspase 9 and caspase 3, and apoptosis [20]. As the apoptosome is the central execution signal downstream of mitochondriaderived death signals, its efficient assembly is imperative for an effective response against drug-induced apoptosis, which invariably involves the intrinsic death pathway. Therefore, we next asked if the resistance of primary BL cells was a function of the lack of cytosolic Apaf-1 expression. To address this, we first made use of our data indicating the ability of the small molecule experimental compounds to induce apoptosis in primary BL cells. Firstly, S-100 cytosol from primary BL cells treated for $1 \mathrm{~h}$ with MPO $(5 \mu \mathrm{M})$, MPO-Zn $(200 \mu \mathrm{M})$, LY30 $(25 \mu \mathrm{M})$ and $\mathrm{NaN}_{3}(5 \mu \mathrm{g} / \mathrm{ml})$ resulted in a significant increase in cell-free caspase 3 activity, indicating efficient apoptosis induction (Figure 2a). Furthermore, while Apaf-1 expression was localized to the $\mathrm{S} 100$ pellets of primary BL cells, exposure of the cells to the novel small molecule compounds resulted in the redistribution of Apaf- 1 to the S100 cytosols (Figure 2b). Of note, caspase 3 activity was also induced in S100 cytosol following treatment of the cells with the cholesterol lowering compound methyl betacyclodextrin (MCD; $200 \mu \mathrm{M})$, also known for its ability

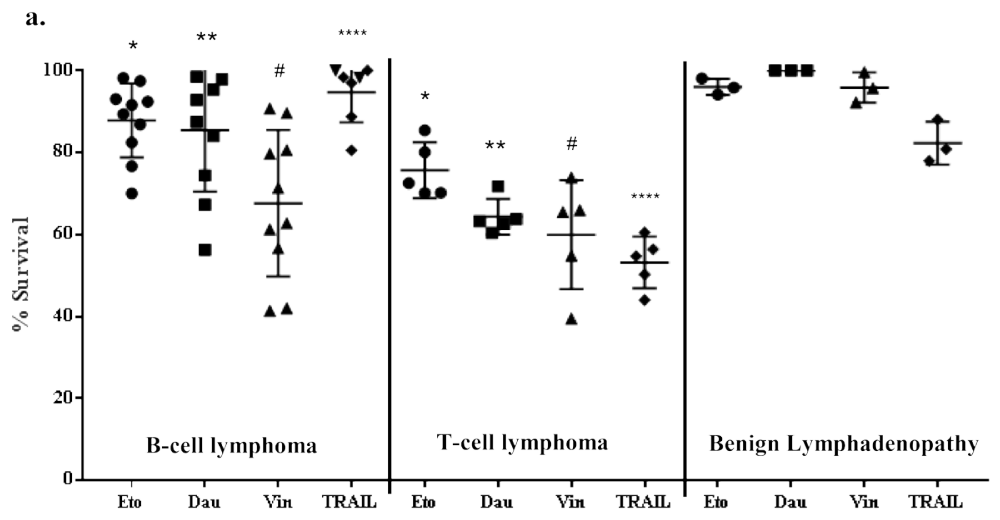

b.
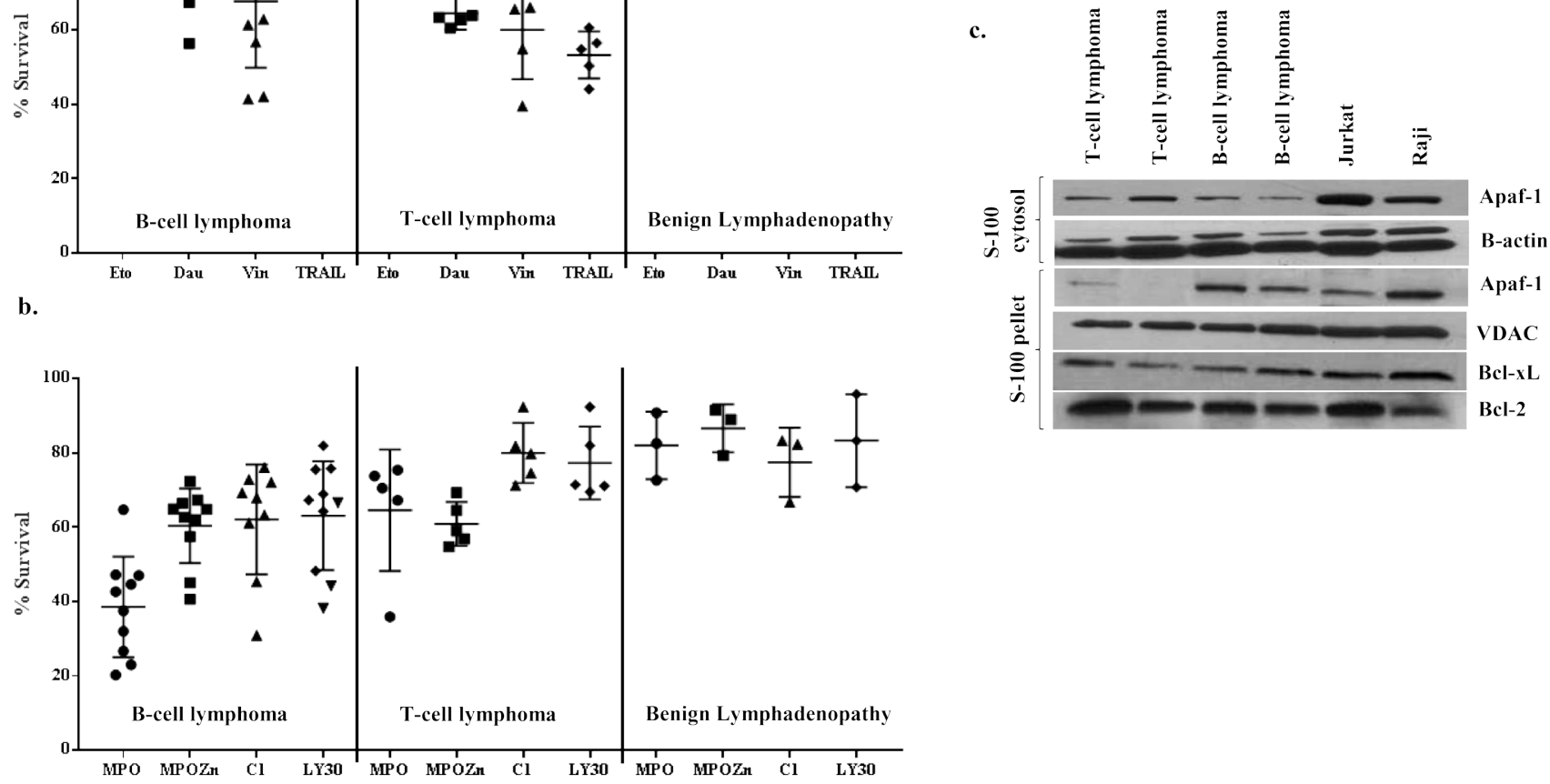

Figure 1: Primary cells from B cell lymphoma patients are resistant to conventional chemotherapeutic agents and contain significantly lower cytosolic Apaf-1: a., b. $2 \times 10^{5}$ primary lymphocytes/200 $\mu 1 /$ well in 96 well plates were exposed to Etoposide $(5 \mu \mathrm{M})$, Daunorubicin $(0.4 \mu \mathrm{g} / \mathrm{ml})$, Vincristine $(5 \mu \mathrm{M}), \mathrm{MPO}(2.5 \mu \mathrm{M}), \mathrm{MPO}-\mathrm{Zn}(200 \mathrm{nM}), \mathrm{C} 1(50 \mu \mathrm{g} / \mathrm{ml}), \mathrm{LY} 30(50 \mu \mathrm{M})$ or TRAIL $(100 \mathrm{ng} / \mathrm{ml})$ for $24 \mathrm{hrs}$. Cell viability was determined by the MTT assay. One way ANOVA multiple comparisons analysis was used for statistical significance $(* p<0.1, * * p<0.001, \# p<0.5, * * * * p<0.0001)$ c. S-100 cytosolic and pellet fractions of primary cells from B-cell and T-cell lymphomas as well as from Raji and Jurkat cell lines were resolved by SDS-PAGE, transferred to PVDF and Apaf-1 was detected by immunoblotting using anti-Apaf-1. Membranes were probed with anti- $\beta$-actin and anti-VDAC-1 as loading controls for cytosolic and pellet fractions, respectively. 
to disrupt membrane lipid raft structures (Figure 2a). To provide conclusive evidence that the cell free system did, indeed, work as expected, we performed additional experiments using the same set of conditions with Raji cells. Results clearly showed minimal caspase 3 activity in the absence of dATP and its robust induction upon addition of dATP (Figure 2c). This was further corroborated by the appearance of Apaf-1 in the cytsolic fractions (Figure 2d). Furthermore, we performed an additional experiment using one of the compounds (MPO) that triggered the relocalization of Apaf-1 to the cytosol in combination with known inducers of apoptosis, etoposide and daunorubicin. Of note, while single agent treatment with etoposide or daunorubicin failed to induce caspase 3 activity in the cellfree model, the presence of MPO significantly enhanced sensitivity to both the agents $(300 \%$ increase in caspase
3 activity as well as increased Apaf-1 release in cytosol (Figure 2e, 2f). These data provide strong evidence that the small molecule compounds facilitate the release of Apaf-1 with the resultant increase in caspase 3 activity.

\section{Aberrant localization of Apaf-1 in the lipid raft fractions of primary BL cells}

Based on the findings that small moleculeinduced sensitization of primary BL cells was linked to the release of Apaf-1 from the membrane fractions to the S100 fractions and our previous study linking apoptosis resistance in B cell lymphoma cell lines to membrane sequestration of Apaf-1 [14], we next assessed the role of the membrane lipid rafts in the resistance

c.

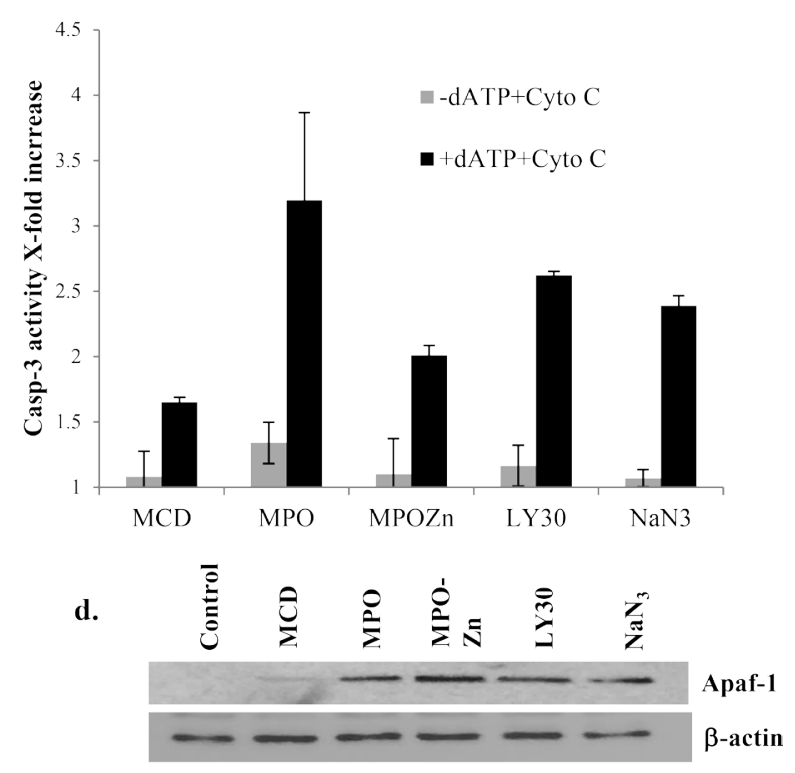

e.
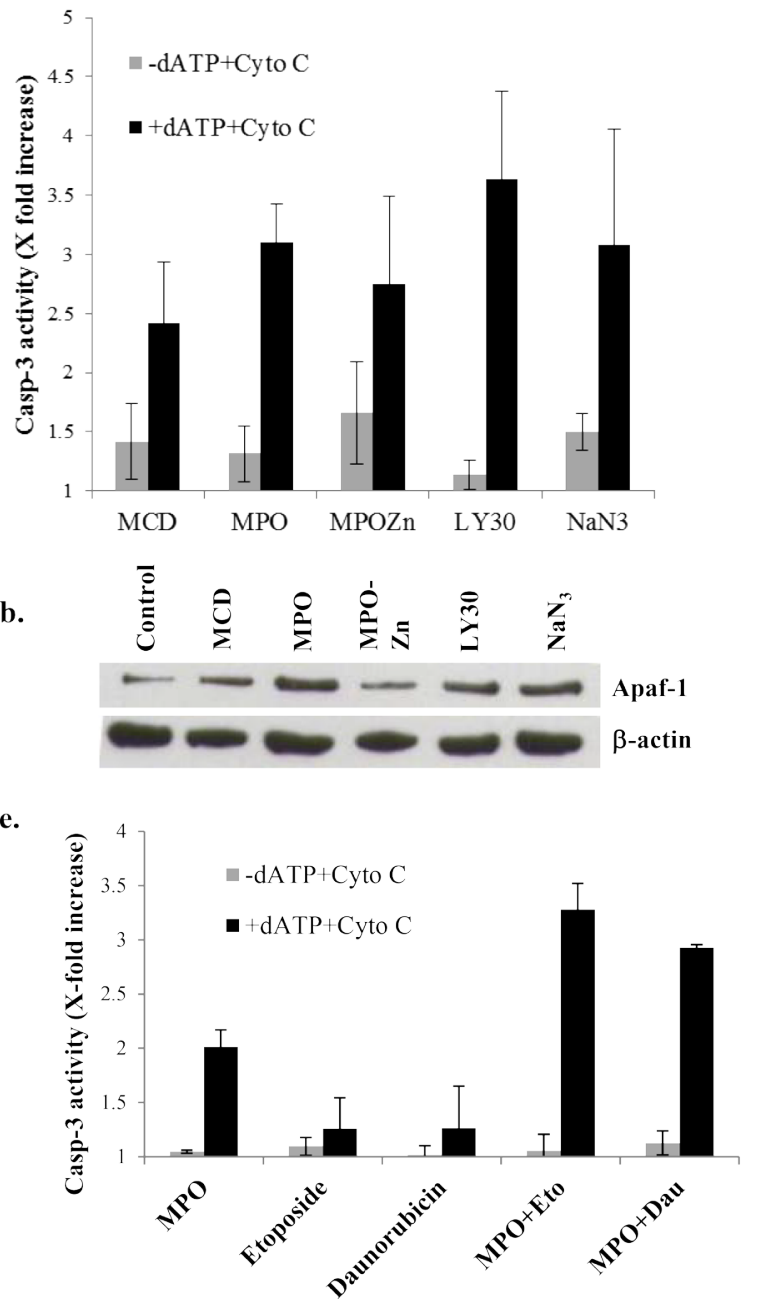

b.

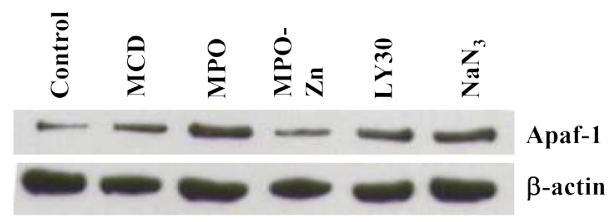

Figure 2: Disruption of Apaf-1 from S-100 pellet increases caspase-3 activity. a., c. S-100 cytosol was prepared from primary BL cells and Raji cells after incubation with MCD $(200 \mu \mathrm{M})$, MPO $(5 \mu \mathrm{M})$, MPO-Zn $(200 \mu \mathrm{M})$, LY30 $(25 \mu \mathrm{M})$ or NaN 3 ( $5 \mu \mathrm{g} / \mathrm{ml})$ for $1 \mathrm{hr}$. Caspase-3 enzyme activity was measured using DEVD-AFC in the treated and untreated S-100 cytosol of B-cell lymphoma in the presence and absence of $1 \mathrm{mM}$ dATP and $4 \mu \mathrm{M}$ cytochrome C. b., d., f. Apaf-1 expression was determined in S-100 cytosol of treated and untreated BL cells by western blotting. Membranes were probed with anti $\beta$-actin for loading control of cytosolic fractions. e. S- 100 cytosolic fraction was purified from Raji cells pre-treated with MPO $(5 \mu \mathrm{M})$ for $1 \mathrm{hr}$ followed by treated with etoposide $(5 \mu \mathrm{M})$ and daunorubicin $(0.4 \mu \mathrm{g} / \mathrm{ml})$. Caspase 3 enzyme activity was then measured in the presence and absence of $1 \mathrm{mM}$ dATP and $4 \mu \mathrm{M}$ cytochrome $\mathrm{c}$. 
of primary BL cells to drug-induced apoptosis. Lipid raft are the cholesterol containing domains within the plasma membrane [21], and the data presented above with the cholesterol lowering compound, MCD, pointed to the possible involvement of the lipid raft domains in the mislocalization of Apaf-1 in primary BL cells. Therefore, membrane fractions were obtained using density gradient centrifugation and subjected to SDSPAGE analysis. Interestingly, in the Raji B cell line, Apaf1 was mostly co-localized with flotillin, which served as the positive control for lipid raft residing proteins (Figure 3a). This was distinctly different from the Jurkat T cell line, where Apaf-1 appeared in the higher cytosolic fractions. Furthermore, treatment with MCD, the lipid raft dissociating agent, resulted in the disruption of the lipid rafts and movement of Apaf-1 to the non-lipid raft fractions of the plasma membrane (Figure 3a). Similar to the effect of MCD, results also provide evidence that the sensitization to apoptosis upon exposure to the small molecule experimental compounds (MPO, MPO$\mathrm{Zn}, \mathrm{C} 1$ and LY30) was also linked to the ability of these compounds to disrupt Apaf-1 from the membrane lipid raft fractions to facilitate efficient assembly and activation of the apoptosome.

Next we assessed whether the mislocalization observed in B cell line Raji was, indeed, a mechanism operative in primary BL cells obtained from patientderived tissue biopsies. Indeed, results obtained on primary BL cells from 10 patient biopsies revealed that Apaf-1 was sequestered in the lipid raft fractions

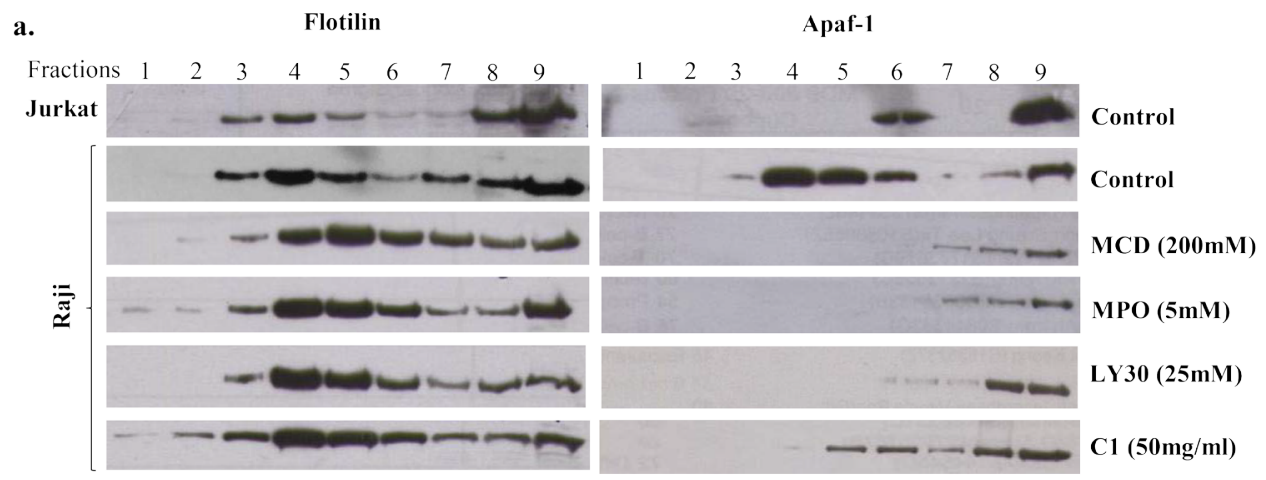

b.

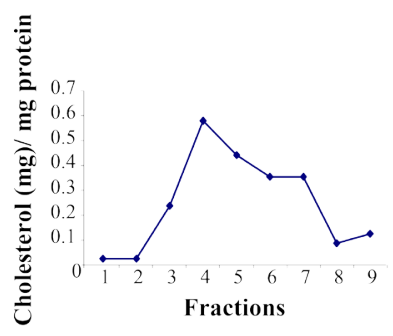

c.

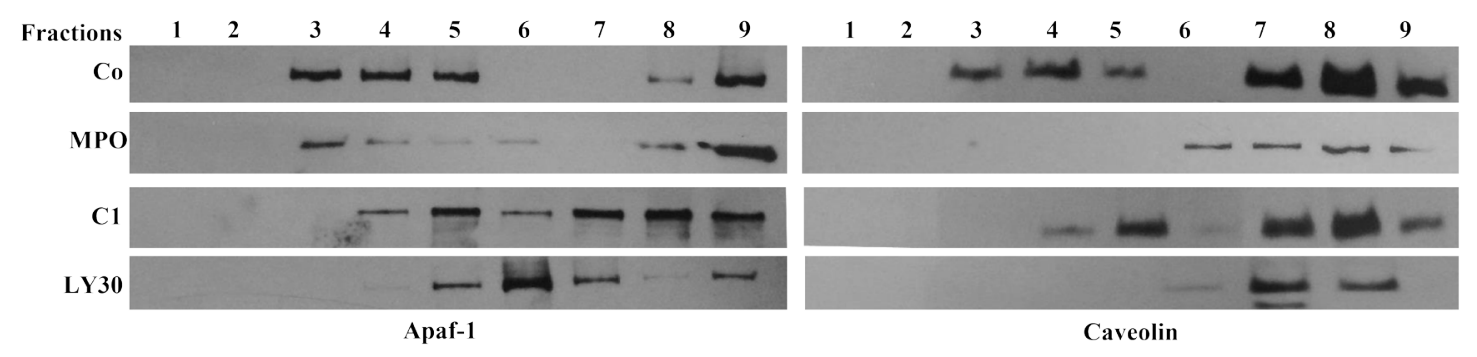

d.

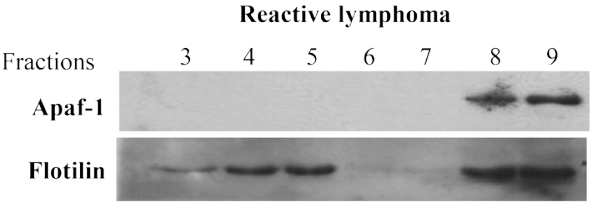

e.

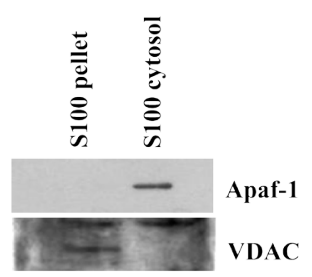

Figure 3: Apaf-1 is sequestered in the lipid raft fractions of Raji cells and primary BL cells. a. Lipid raft fractions were prepared from Jurkat and Raji cells as described in Material and Methods. Raji cells were treated with MCD $(200 \mu \mathrm{M}), \mathrm{MPO}(5 \mu \mathrm{M}), \mathrm{C} 1$ $(50 \mu \mathrm{g} / \mathrm{ml})$ and LY30 $(25 \mu \mathrm{M})$ for $1 \mathrm{hr}$ before processing for lipid raft fractions. Apaf-1 expression was assessed in the different raft fractions by western blotting. Flotillin was used as a positive control for lipid raft fractions. b. Cholesterol content was determined in each lipid raft fraction according to manufacturer's instructions as described in Materials and Methods. c. Lipid raft fractions were prepared from primary cells derived from B-cell lymphoma and reactive lymphadenitis, as described in Material and Methods. Primary BL cells were treated with MPO $(5 \mu \mathrm{M}), \mathrm{C} 1(50 \mu \mathrm{g} / \mathrm{ml})$, or LY30 $(25 \mu \mathrm{M})$ for $1 \mathrm{hr}$ before processing for lipid raft fractions. Apaf-1 expression was checked in all the raft fractions by Western blotting, Caveolin was used as a positive control for lipid raft fractions. d. For reactive lymphadenitis, Apaf-1 was assessed in all raft fractions by western blotting. Flotillin was used as a positive control for lipid raft fractions. e. S-100 cytosol and pellets were prepared from primary cells from reactive lymphadenitis and Apaf-1 expression was detected by Western blotting. 
and that treatment of the cells with the small molecule experimental compounds MPO $(5 \mu \mathrm{M})$, LY30 $(25 \mu \mathrm{M})$, $\mathrm{C} 1(50 \mu \mathrm{g} / \mathrm{ml})$ for $1 \mathrm{hr}$, resulted in a significant decrease in the localization of Apaf-1 to the lipid raft fractions (Figure 3c, Supplementary Figure 2). To verify that sequestration of Apaf-1 was specifically limited to B-cell lymphomas, biopsies from patients with noncancerous reactive lymphadenopathy were used. Notably, Apaf-1 was not associated with the lipid raft fractions (Figure 3d) but expressed in the S100 cytosol fractions (Figure 3e) in cells derived from non-cancerous lesions. Furthermore, we determined the cholesterol content of the fractions to validate the identity of the fractions, given the observations that these sub-domains are enriched in sphingolipids and cholesterol [22]. Indeed, fractions 3, 4 and 5 that are indicative of lipid raft fractions did show the highest level of cholesterol (Figure 3b). To further validate these findings, the same patient material was subjected to immunohistochemistry for the expression of Apaf-1. Indeed, Apaf-1 co-localized with the lipid raft marker protein caveolin (Figure 4), thereby corroborating the data presented in the preceding sections. Taken together, Apaf1 mislocalization to lipid raft domains is a critical factor in apoptosis resistance in clinical B-cell lymphomas and strategies to disrupt these cholesterol-rich domains appear to restore death sensitivity.

\section{Small molecule induced re-localization of Apaf-1 to the cytosol is ROS dependent}

We were intrigued by the ability of the small molecule experimental compounds to not only dislodge Apaf-1 from the membrane lipid raft-like structures, but also to restore the sensitivity to apoptosis. Two of the molecules (LY30 and C1) have been previously reported by our group to target tumor cells via mechanisms involving an increase in intracellular $\operatorname{ROS}[15-17,23]$. As ROS have been linked to membrane lipid peroxidation and disruption of lipid rafts, we asked if the ability of
C1 and LY30 to dislodge Apaf-1 from the lipid rafts was a function of intracellular ROS production. Indeed, exposure of Raji B cells to C1 $(50 \mu \mathrm{g} / \mathrm{ml})$ or LY30 $(25 \mu \mathrm{M})$ resulted in a significant increase in intracellular ROS production measured by the change in DCF oxidation (Figure 5a). Furthermore, pre-incubation with 3000 Units of catalase significantly attenuated the increase in fluorescence, thereby indicating the involvement of hydrogen peroxide $\left(\mathrm{H}_{2} \mathrm{O}_{2}\right)$. More importantly, both $\mathrm{C} 1$ and LY30 induced a change in the localization of Apaf1 in the membrane fractions (fraction 5 as compared to fractions 4 and 5 that co-localize with flotillin), which was reversed upon a priori treatment with catalase (Figure 5b), further supporting the involvement of $\mathrm{H}_{2} \mathrm{O}_{2}$. Interestingly, not only has $\mathrm{H}_{2} \mathrm{O}_{2}$ been directly implicated in lipid peroxidation [24], but also ROS-induced lipid peroxidation has been linked to the modification of membrane lipid rafts [25]. Indeed, we provide evidence that treatment with the ROS producing compound C1 (25 and $50 \mu \mathrm{g} / \mathrm{ml}$ for $15-60$ minutes) resulted in an increase in membrane lipid peroxidation, which was prevented by the $\mathrm{H}_{2} \mathrm{O}_{2}$ scavenger catalase (Figure 5c). Taken together, these data indicate that ROS generating agents could serve as an excellent tool for the re-localization of Apaf-1 to the cytosol in B cell lymphomas that are rendered refractory to apoptosis due to the membrane sequestration of this essential adaptor protein

Next, we undertook expression profiling to identify specific gene signatures and/or gene marker(s) differentially expressed in lymphomas with membrane localized Apaf-1. To check gene expression, RNA was extracted from same patients' biopsies and analyses were performed. Briefly, 186 probe sets were differentially expression by 2 fold between samples with membrane APAF1 and those with cytosolic APAF1. 132 probe sets were over-expressed and 64 under-expressed. Only probe sets of coding genes and those that are differentially expressed, also at a significance of $\mathrm{p}<0.05$, are represented on the heat map (Figure 6a). Interestingly, BCL11A was amongst the genes over-expressed in the samples with
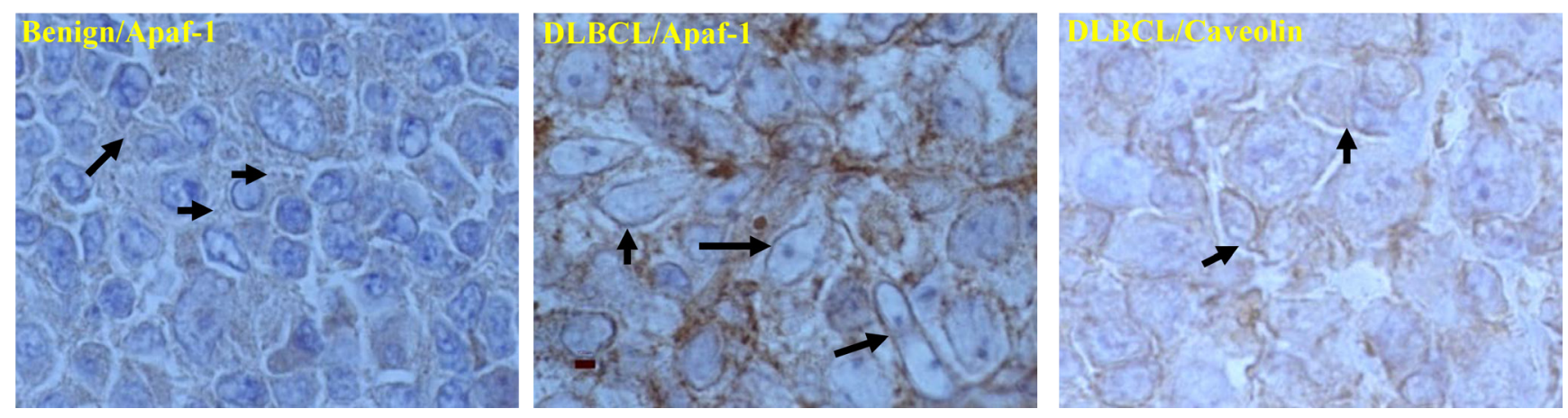

Figure 4: Co-localization of Apaf-1 and caveolin (lipid raft marker) in B-cell Lymphoma. Apaf-1 distribution is shown in both benign and DLBCL by immunohistochemistry. Benign and DLBCL section slides were de-paraffinized and stained with anti-Apaf-1HRP or anti-Caveolin-HRP, followed by secondary anti-HRP. 
membrane APAF1. The expression of BCL11A was further validated at the protein level by western blot in the same patient's biopsy material (Figure 6b). These data, albeit with a small number of samples, point to a possible correlation between membrane localization of Apaf-1 and BCL11A overexpression [26, 27], which could serve as novel indicator of drug resistance or poor prognosis in $B$ cell lymphomas.

\section{DISCUSSION}

DLBCL is the most common type of B-cell lymphoma that exhibits drug resistant phenotype and a high incidence of relapse [1]. As most chemotherapeutic agents preferentially activate mitochondria-driven death execution via mechanisms that trigger the induction of
MOMP [2], the major focus in terms of the acquisition of drug resistance in the clinical setting has been on the anti-apoptotic members of the Bcl-2 family, such as Bcl2 and Bcl-xL [28]. However, there is also accumulating evidence that a defect in post-MOMP signaling stalls death execution and contributes to the development of the resistant phenotype [3, 29]. In this regard, we previously reported a novel mechanism for the defective apoptosis signaling in the human B cell line, Raji, whereby defective apoptosome formation due to the absence of cytosolic Apaf-1 was identified as a critical factor [14]. In the present study, we provide clinical evidence that mislocalization of Apaf-1 may represent an important prognostic factor in the therapeutic management of DLBCL.

Apaf-1 is an essential adaptor protein, which serves

a.

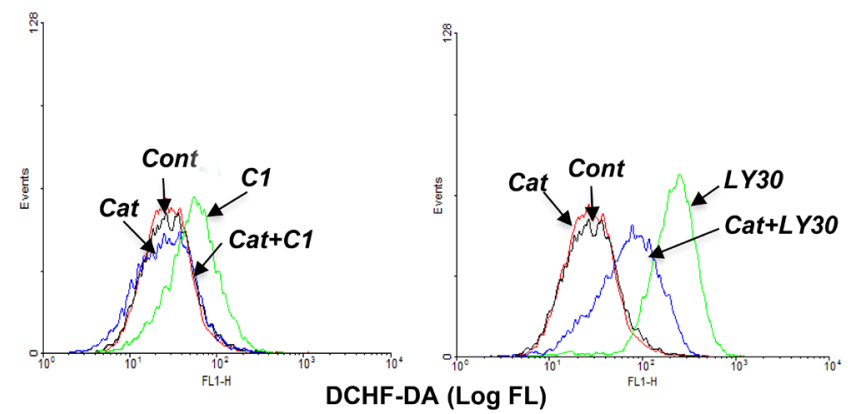

b.

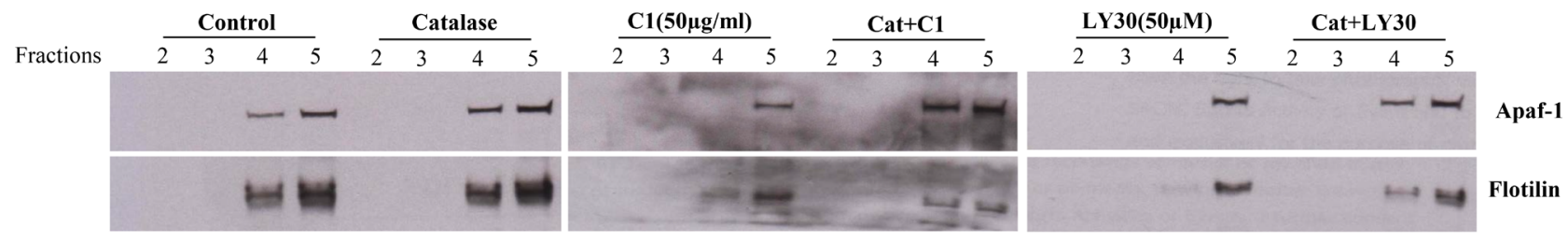

c.

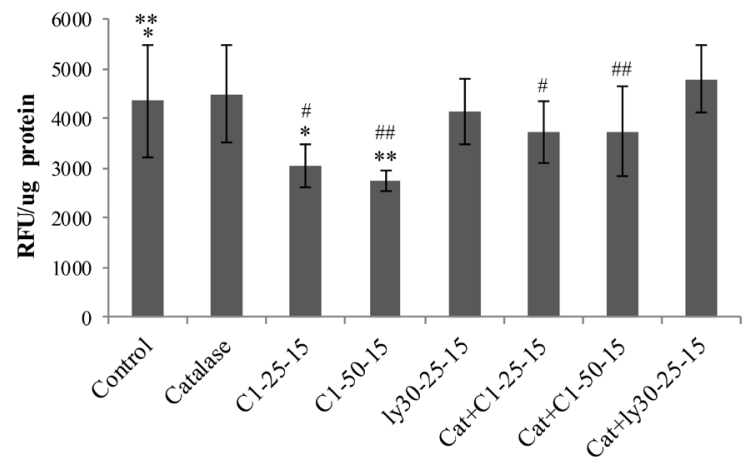

Figure 5: Small molecule-induced re-distribution of Apaf-1 to the cytosol is ROS dependent. a. Raji cells were preincubated with Catalase $(3000 \mathrm{U} / \mathrm{ml})$ followed by incubation with $\mathrm{C} 1(50 \mu \mathrm{g} / \mathrm{ml})$ or LY30 $(25 \mu \mathrm{M})$ for $1 \mathrm{hr}$. Intracellular ROS was assessed using the fluorescent probe DCHF-DA as described in Materials and Methods. b. Lipid raft fractions were purified from the same cells and Apaf-1 expression was checked in fractions 2, 3, 4 and 5 by Western blotting. Flotillin was used as a control for lipid raft resident protein. c. Raji cells were pre-incubated with catalase $(3000 \mathrm{U} / \mathrm{ml})$ followed by exposure to C1 $(25,50 \mu \mathrm{g} / \mathrm{ml})$ or LY30 $(25,50 \mu \mathrm{M})$ for 15,30 and 60 min. Lipid peroxidation was assessed using the fluorescence based assay using Cis-perinaric acid as a probe. $*, * *, \#, \# \# p<0.05$ 
as the scaffold for the recruitment of cytochrome C, procaspase 9, and dATP, to bring about the assembly of the apoptosome, and activation of downstream caspase 9 activation[4]. In healthy cells, Apaf-1 exists in the cytosol [30] as a monomer in a complex with dATP/ ATP, a conformation that prevents its oligomerization and downstream recruitment of pro-caspase 9. Interestingly, loss of Apaf-1 has been reported in a variety of human cancers, such as melanoma, leukemia, ovarian carcinoma, bladder carcinoma and glioblastoma [8, 10, 13, 31]. Similarly, the development of resistance to death receptorand drug-induced apoptosis in colon carcinoma was associated with downregulation of Apaf-1 [32]. Our results not only corroborate the critical importance of functional Apaf-1 in DLBCL, but also provide a novel mechanism for its loss of function. While the overall expression of Apaf-1 did not change in the clinical lymphomas we examined, the sub-cellular localization was significantly altered such that the protein was sequestered in lipid raft domains of the plasma membrane instead of being expressed in the cytosol. The importance of this mislocalization in the context of apoptosis resistance (ex vivo) was further corroborated by results demonstrating the restoration of caspase 3 cleavage/activation upon cytosolic redistribution of Apaf-1.

Lipid rafts are cholesterol-rich structural domains within the plasma membrane, which contain diverse protein and lipid components. Interestingly, membrane rafts are actively involved in the maintenance and regulation of signal transduction, as a number of essential signaling proteins have been shown to localize to these sub-domains, such as recruitment of receptors and/ or their ligands to the rafts to facilitate intracellular signaling [22]. By the same token rafts could interfere

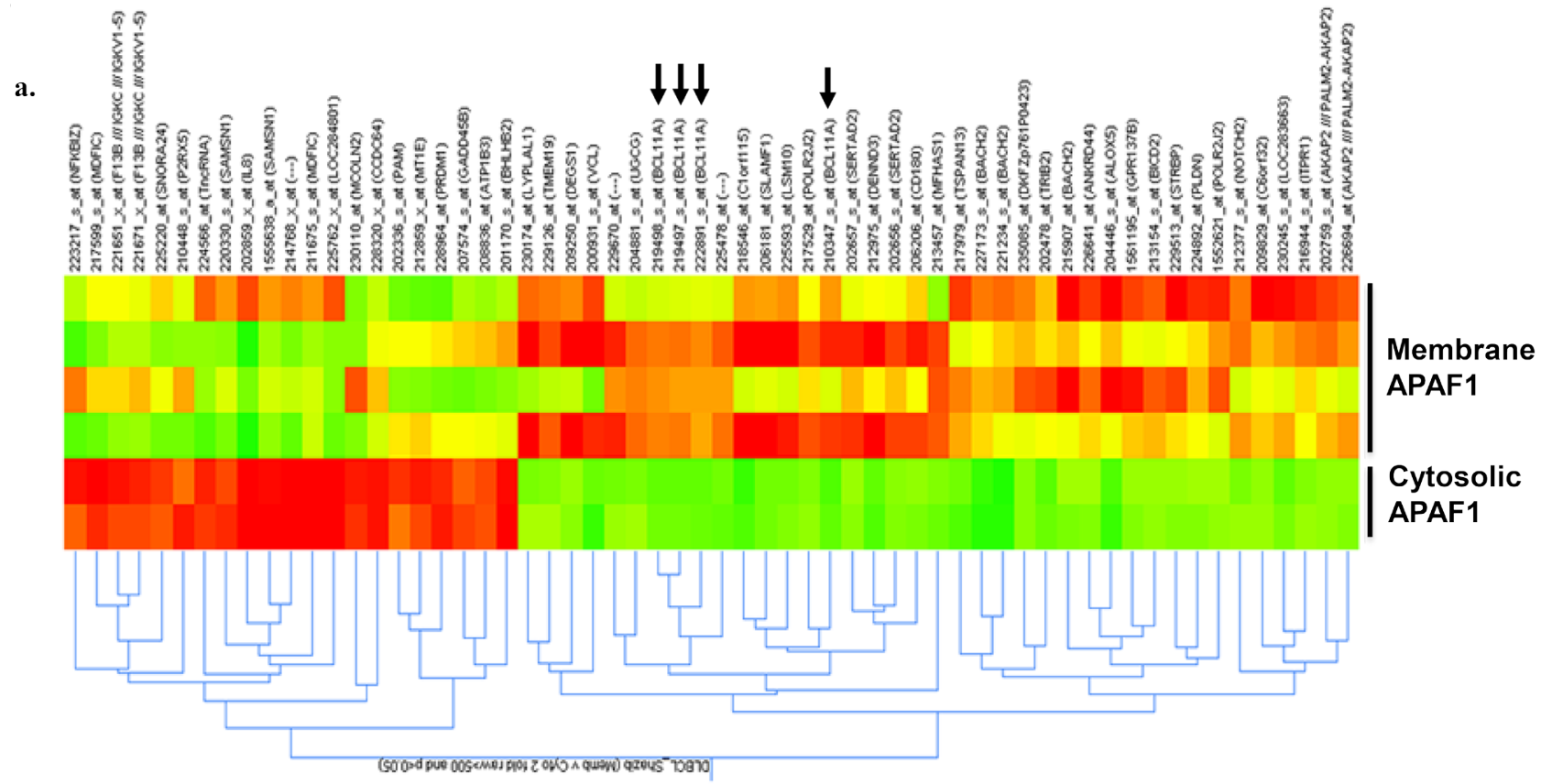

b.

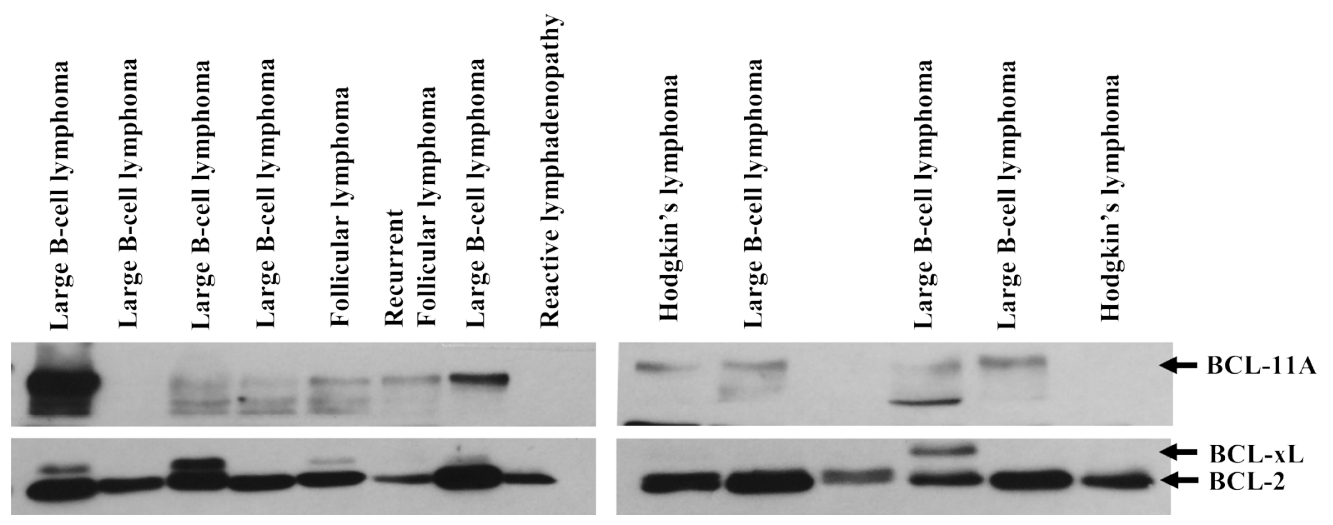

Figure 6: Higher expression of BCL11A in B-cell Lymphoma. a. Gene expression profiling and analysis was done using Affymetrix HG-U133 Plus 2 microarray chips. Differentially expressed genes were selected based on mean expression intensity of 500, and $>2$ fold difference in expression between samples with membrane APAF1 location versus samples with Cytosolic APAF1 location. b. Total lysates of primary cells from the same lymphoma tissues were prepared and the expression of BCL11A was assessed by western blotting. 
or block cell signaling by sequestering proteins away from a specific signaling network. Of note, membrane rafts have been shown to play an important role in the activation and regulation of $\mathrm{B}$ cell receptor-mediated signaling [33]. Furthermore, critical components of $\mathrm{B}$ cell receptor signaling are absent from lipid rafts in certain disease states involving B cells, such as the EBV proteins LMP-1 and LMP-2A. While LMP-1 is involved in the sustained proliferative signal downstream of $\mathrm{B}$ cell receptor activation [34], LMP-2A sequesters Syk and Lyn tyrosine kinases away from the B cell receptor to block signal transduction [35]. These data seem to imply that recruitment to the lipid raft domains may not be exclusively restricted to membrane proteins or proteins with lipid-anchoring moieties. Such appears to be the case with Apaf-1, a predominantly cytosolic protein, but shown here to be sequestered in lipid raft fractions of DLBCL cells, thereby creating a non-permissive environment for apoptosome assembly and activation. The critical importance of this mislocalization of Apaf-1 in the overall biology of DLBCL is further supported by our results demonstrating the ability of small molecules that disrupt raft-like domains via their effect on cholesterol (MCD) or lipid peroxidation via ROS production (Ly30 and C1); however, the precise mechanism underlying the lipid raft recruitment of Apaf-1 is still the focus of our ongoing investigations. One could conjecture the possibility of an interaction of Apaf-1 with lipid raft-associated proteins, such as the receptor tyrosine kinase, c-Met, which is used as a prognostic marker for DLBCL [36]. Moreover, as EBV infection is a frequent finding in patients with $B$ cell lymphoma [37], the possibility of viral proteins, such as LMP1, hijacking Apaf-1 to the lipid fractions cannot be completely ruled out. Similarly, the existence of an interaction between the WD40 domain within Apaf-1 [38] and membrane associated cytoskeleton protein(s) could be another plausible scenario. Furthermore, gene expression data also point to an interesting association between membrane-anchored Apaf-1 and overexpression of the Kruppel zinc finger gene, BCL11A. It should be pointed out that overexpression of BCL11A has previously been associated with hematopoietic malignancies, either through its gene amplification or chromosomal translocation [26, 27]. The gene expression data were confirmed at the protein level; however, these observations need to be validated in a larger cohort of patients to gain further insight into the functional relevance of the association between membrane localized Apaf-1 and BCL11A expression. Nevertheless, the preliminary association lends itself to suggest a possible marker of drug resistance and/or poor prognosis in B cell lymphomas.

One other attractive model proposed earlier by Kagan et al. surmised that the significant differences in the membrane composition as well as the levels of ROS in B-cell lymphomas, compared to their $\mathrm{T}$ cell counterparts, may affect the protein and cholesterol trafficking in the cells [39]. Although, we do not have experimental evidence to suggest differences in the membrane composition between primary cells derived from B and T cell lymphomas, results presented with small molecule experimental compounds appear to support these observations. Two small molecule experimental agents, $\mathrm{C} 1$ $[16,17]$ and LY30 $[15,23]$, as well as the mitochondria electron transport inhibitor $\mathrm{NaN}_{3}$ that trigger intracellular ROS production had a profound influence on cell-free caspase 3 activity as a result of the re-localization of Apaf1 to the cytosol. To that end, redox-dependent alterations of lipid moieties could disrupt lipid raft structures thereby facilitating the release of sequestered Apaf-1. This is supported, at least in part, by the inhibitory effect of scavenging intracellular $\mathrm{H}_{2} \mathrm{O}_{2}$ with catalase. Collectively, these data suggest a novel mechanism for reinforcing apoptososme activation in DLBCL via drug-induced cytosolic redistribution of the apoptosome scaffold protein, Apaf-1, which is trapped in membrane raft sub-domains in refractory DLBCL.

\section{CONCLUDING REMARKS:}

Primary cells derived from patients with DLBCL show membrane raft sequestration of the apoptosome adaptor protein, Apaf-1. As Apaf-1 expression is critical for post-mitochondrial death execution, these data provide a novel mechanism of the refractory and aggressive nature of this sub-class of B cell lymphomas. Recent efforts have been geared at identifying novel agents that facilitate the robust assembly of apoptosome components and the major focus has been on compounds that promote the egress of cytochrome C, an essential apoptosome protein. Our data provide a novel strategy whereby the redistribution of Apaf-1 to the cytosol via drugs that act on lipid raft sub-domains through distinct mechanisms, including alterations in intracellular redox milieu, could create a favorable environment for apoptosome activation and death execution with potential therapeutic implications for the management of refractory DLBCL.

\section{MATERIALS AND METHODS}

\section{Primary lymphoma cells and cancer cell lines}

Tissue biopsies from lymphoma patients were obtained from National University Hospital (NUHS) of Singapore after obtaining proper informed consent from the patients. Tissues were minced and strained using MACS separation filter (Milteny Biotec, Bergisch Gladbach, Germany) to obtain a singlecell suspension. Few samples were sorted out using CD22 and CD8 antibodies by magnetic cell sorting according to manufacturer's protocol (Milteney Biotec, 
Bergisch Gladbach, Germany). Mononuclear cells and/ or lymphocytes' enrichment was performed by Ficoll Hypaque (Sigma, St Louis, MO) gradient centrifugation. A total of 58 tissue biopsies were obtained from consenting patients (37 B cell lymphomas, $9 \mathrm{~T}$ cell lymphomas and 12 benign tumors) and used in this study. For in vitro validation, Raji (Burkitt's lymphoma) and Jurkat (T-cell Lymphoma) human cell lines were cultured in RPMI1640 supplemented with 10\% FBS, 1\% L-glutamine, 1\% penicillin and streptomycin in a humidified incubator with $5 \% \mathrm{CO}_{2}$.

\section{Determination of cell viability}

Cell viability was determined by the MTT assay after exposure of $2 \times 10^{5}$ primary lymphocytes/200 $\mu \mathrm{l} /$ well in 96 well plates to etoposide $(5 \mu \mathrm{M})$, daunorubicin $(0.4 \mu \mathrm{g} / \mathrm{ml})$, vincristine $(5 \mu \mathrm{M})$ (Sigma, St Louis, MO), mercaptopyridine oxide (MPO; 2.5 $\mathrm{M}$ ), MPO-Zn (200nM), C1 $(50 \mu \mathrm{g} / \mathrm{ml})$, LY303511 (Calbiochem, Billerica, MA) (LY30; 50 $\mu \mathrm{M})$ and TRAIL (100ng/ml) for $24 \mathrm{hrs}$. MTT assay was performed as described previously [40]. To evaluate the ability of the small molecule compounds to sensitize to death receptor-induced apoptosis, $2 \times 10^{5}$ primary lymphocytes/200 $\mu 1 /$ well in 96 well plates were pretreated for $1 \mathrm{hr}$ with MPO $(0.625 \mu \mathrm{M})$, MPO-Zn $(50 \mathrm{nM}), \mathrm{C} 1(25 \mu \mathrm{g} / \mathrm{ml})$ or LY30 $(25 \mu \mathrm{M})$ prior to the addition of TRAIL $(50 \mathrm{ng} / \mathrm{ml})$ for $24 \mathrm{hrs}$ and cell viability was determined by the MTT assay.

\section{Assessment of cell-free caspase-3 activity}

For S-100 cytosolic extraction, $30 \times 10^{6}$ cells (primary lymphocytes, Raji or Jurkat) were incubated with ice-cold S-100 buffer (20mM HEPES-KOH at $\mathrm{pH} 7.5$, $10 \mathrm{mM} \mathrm{KCL}, 1.5 \mathrm{mM} \mathrm{MgCl}{ }_{2}, 1 \mathrm{mM}$ EDTA, $1 \mathrm{mM}$ EGTA, $1 \mathrm{mM}$ DTT supplemented with protease inhibitors and phosphatase inhibitors (Sigma, St Louis, MO). Cells were disrupted using a Dounce homogenizer and the pellet was centrifuged at $1000 \mathrm{~g}$ for 10 minutes at $4^{\circ} \mathrm{C}$. Supernatants were further centrifuged at $100,000 \mathrm{~g}$ for $1 \mathrm{hr}$, and the resulting supernatant $\left(\mathrm{S}-100\right.$ cytosol) was stored at $-80^{\circ} \mathrm{C}$ or used immediately. The S-100 pellet was dissolved in 1X RIPA lysis buffer with protease and phosphatase inhibitors and stored at $-80^{\circ} \mathrm{C}$ or used immediately. For

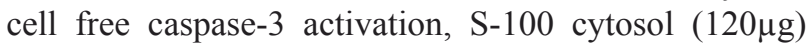
from cells treated with MPO $(5 \mu \mathrm{M}), \mathrm{MPO}-\mathrm{Zn}(200 \mu \mathrm{M})$, LY30 $(25 \mu \mathrm{M}), \mathrm{NaN}_{3}(5 \mu \mathrm{g} / \mathrm{ml})$, etoposide $(5 \mu \mathrm{M})$ or daundorubicin $(0.5 \mu \mathrm{l} / \mu \mathrm{l})$ were incubated in the presence or absence of $1 \mathrm{mM}$ dATP and $4 \mu \mathrm{M}$ cytochrome C (Sigma, St Louis, MO) for $30 \mathrm{~min}$ at $37^{\circ} \mathrm{C}$. Caspase- 3 activity was then measured by using fluorogenic substrate DEVD-AFC as described previously [40].

\section{Western blot analysis}

S-100 cytosol and pellet fractions or lipid raft fractions were denatured in loading buffer and resolved on $10 \%$ sodium dodecyl sulfate-polyacrylamide gel electrophoresis (SDS-PAGE) followed by transfer to PVDF membranes. Immunoblotting was performed with antibodies against Apaf-1 (Chemicon, CA), $\beta$-actin, Bcl2, Bcl-xl, VDAC-1 (Santa-Cruz, Dallas, TX), BCL11A, caveolin and flotillin (Abcam, Cambridge, UK) and then probed with the isotype specific secondary IgG-HRP as required. Membranes were then exposed to Super Signal Substrate Western Blotting Kit (Pierce, Rockford, IL, USA).

\section{Purification of membrane lipid raft fraction}

For lipid raft extraction, $40 \times 10^{6}$ cells (primary lymphocytes, Raji or Jurkat) were incubated in icecold HEPES buffer (pH 7.4) for 20 minutes. Cells were disrupted using a Dounce homogenizer followed by sonication and the lysates were centrifuged at $1000 \mathrm{~g}$ for 10 minutes at $4^{\circ} \mathrm{C}$. The resulting supernatant was loaded onto a gradient of $80 \%, 30 \%$, and $5 \%$ sucrose and centrifuged at $38000 \mathrm{RPM}$ for 21 hours at $4^{\circ} \mathrm{C} .500 \mathrm{ul}$ of the fraction was collected from the top and stored at $-80^{\circ}$ or used immediately. Fractions were used for the determination of cholesterol content using the cholesterol quantitation kit (Calbiochem, Billerica, MA) following the manufacturers recommended protocol, as well as subjected to SDS-PAGE for western blot analysis of membrane proteins.

\section{Determination of intracellular ROS and lipid peroxidation}

$2 \times 10^{6}$ primary lymphocytes or Raji cells were treated with or without $\mathrm{C} 1(25$ and $50 \mu \mathrm{g} / \mathrm{ml})$ or LY30 $(25$ and $50 \mu \mathrm{M})$ for 15,30 and 60 minutes. Cells were incubated with catalase $(3000 \mathrm{U} / \mathrm{ml})$ one hour prior to the addition of the drugs and cellular ROS content was measured by loading cells with DCHF-DA (Molecular Probes, Eugene, OR), as described previously [23]. For the assessment of lipid peroxidation, samples were incubated with cis-perinaric acid $(10 \mu \mathrm{M})$ (Sigma, St Louis, MO) for $1 \mathrm{hr}$ at $37^{\circ} \mathrm{C}$, and a change in the fluorescence emission at $418 \mathrm{~nm}$ following excitation at $324 \mathrm{~nm}$ was used to indicate an increase in lipid peroxidation.

\section{Immunohistochemistry}

Formalin-fixed paraffin embedded lymphoma sections were obtained from National University Hospital Singapore, Singapore. The slides were de-paraffinized and endogenous peroxidase activity was inhibited with 
$3 \%$ hydrogen peroxide in $1 \mathrm{X}$ PBS. Sections were stained with HRP-conjugated primary antibody for overnight followed by the secondary anti-HRP antibody. DAB substrate (DAKO, Denmark) was added for $5 \mathrm{~min}$ at room temperature before washing slides with distilled water. Slides were counterstained with hematoxylin for $5 \mathrm{sec}$ before being mounted with aqueous mounting media and viewed under a light microscope.

\section{Gene expression profiling and analysis}

Gene expression microarray was performed using Affymetrix HG-U133 Plus 2 microarray chips. CEL files were processed using the MAS5 algorithm. Differentially expressed genes were selected based on mean expression intensity of 500, and $>2$ fold difference in expression between samples with membrane APAF1 location versus samples with Cytosolic APAF1 location with t-test p-value of 0.05 .

\section{ACKNOWLEDGMENTS}

This work was supported by grants from the National Medical Research Council, Singapore (NMRC/0738/2003) and Singapore Cancer Syndicate (SCS-NPU94R) to S.P. Special thanks to Karishma Sachaphibulkij for her contributions to Figure $3 \mathrm{a}$ and Patricia Pei Wen Tay for helping out with Supplementary Figure 1a and 1c.

\section{CONFLICTS OF INTEREST}

The Authors do not have any potential conflict of interest to disclose.

\section{REFERENCES}

1. Habermann TM, Weller EA, Morrison VA, Gascoyne RD, Cassileth PA, Cohn JB, Dakhil SR, Woda B, Fisher RI, Peterson BA and Horning SJ. Rituximab-CHOP versus CHOP alone or with maintenance rituximab in older patients with diffuse large B-cell lymphoma. J Clin Oncol. 2006; 24(19):3121-3127.

2. Tait SW and Green DR. Mitochondria and cell death: outer membrane permeabilization and beyond. Nat Rev Mol Cell Biol. 2010; 11(9):621-632.

3. Fadeel B, Ottosson A and Pervaiz S. Big wheel keeps on turning: apoptosome regulation and its role in chemoresistance. Cell Death Differ. 2008; 15(3):443-452.

4. Riedl SJ and Salvesen GS. The apoptosome: signalling platform of cell death. Nat Rev Mol Cell Biol. 2007; 8(5):405-413.

5. Hill MM, Adrain C, Duriez PJ, Creagh EM and Martin SJ. Analysis of the composition, assembly kinetics and activity of native Apaf-1 apoptosomes. EMBO J. 2004; 23(10):2134-2145.

6. Kim HE, Du F, Fang $M$ and Wang X. Formation of apoptosome is initiated by cytochrome c-induced dATP hydrolysis and subsequent nucleotide exchange on Apaf-1. Proc Natl Acad Sci U S A. 2005; 102(49):17545-17550.

7. Hajra KM and Liu JR. Apoptosome dysfunction in human cancer. Apoptosis. 2004; 9(6):691-704.

8. Soengas MS, Capodieci P, Polsky D, Mora J, Esteller M, Opitz-Araya X, McCombie R, Herman JG, Gerald WL, Lazebnik YA, Cordon-Cardo C and Lowe SW. Inactivation of the apoptosis effector Apaf-1 in malignant melanoma. Nature. 2001; 409(6817):207-211.

9. $\mathrm{Fu}$ WN, Bertoni F, Kelsey SM, McElwaine SM, Cotter FE, Newland AC and Jia L. Role of DNA methylation in the suppression of Apaf-1 protein in human leukaemia. Oncogene. 2003; 22(3):451-455.

10. Jia L, Srinivasula SM, Liu FT, Newland AC, FernandesAlnemri T, Alnemri ES and Kelsey SM. Apaf-1 protein deficiency confers resistance to cytochrome c-dependent apoptosis in human leukemic cells. Blood. 2001; 98(2):414421.

11. Allen JD, Zhang XD, Scott CL, Boyle GM, Hersey P and Strasser A. Is Apaf-1 expression frequently abrogated in melanoma? Cell Death Differ. 2005; 12(6):680-681.

12. Christoph F, Kempkensteffen C, Weikert S, Kollermann J, Krause H, Miller K, Schostak M and Schrader M. Methylation of tumour suppressor genes APAF-1 and DAPK-1 and in vitro effects of demethylating agents in bladder and kidney cancer. Br J Cancer. 2006; 95(12):17011707.

13. Leo C, Richter C, Horn LC, Schutz A, Pilch H and Hockel M. Expression of Apaf-1 in cervical cancer correlates with lymph node metastasis but not with intratumoral hypoxia. Gynecol Oncol. 2005; 97(2):602-606.

14. Sun Y, Orrenius S, Pervaiz S and Fadeel B. Plasma membrane sequestration of apoptotic protease-activating factor-1 in human B-lymphoma cells: a novel mechanism of chemoresistance. Blood. 2005; 105(10):4070-4077.

15. Shenoy K, Wu Y and Pervaiz S. LY303511 enhances TRAIL sensitivity of SHEP-1 neuroblastoma cells via hydrogen peroxide-mediated mitogen-activated protein kinase activation and up-regulation of death receptors. Cancer Res. 2009; 69(5):1941-1950.

16. Wong $\mathrm{CH}$, Iskandar KB, Yadav SK, Hirpara JL, Loh T and Pervaiz S. Simultaneous induction of non-canonical autophagy and apoptosis in cancer cells by ROS-dependent ERK and JNK activation. PLoS One. 2010; 5(4):e9996.

17. Iskandar K, Rezlan M, Yadav SK, Foo CH, Sethi G, Qiang Y, Bellot GL and Pervaiz S. Synthetic Lethality of a Novel Small Molecule Against Mutant KRAS-Expressing Cancer Cells Involves AKT-Dependent ROS Production. Antioxidants \& Redox Signaling. 2016; 24(14): 781-94.

18. Holme AL, Yadav SK and Pervaiz S. Automated laser 
scanning cytometry: a powerful tool for multi-parameter analysis of drug-induced apoptosis. Cytometry A. 2007; 71(2):80-86.

19. Testa U. TRAIL/TRAIL-R in hematologic malignancies. J Cell Biochem. 2010; 110(1):21-34.

20. Schafer ZT and Kornbluth S. The apoptosome: physiological, developmental, and pathological modes of regulation. Dev Cell. 2006; 10(5):549-561.

21. Jacobson K, Mouritsen OG and Anderson RG. Lipid rafts: at a crossroad between cell biology and physics. Nat Cell Biol. 2007; 9(1):7-14.

22. Pike LJ. Lipid rafts: bringing order to chaos. J Lipid Res. 2003; 44(4):655-667.

23. Poh TW and Pervaiz S. LY294002 and LY303511 sensitize tumor cells to drug-induced apoptosis via intracellular hydrogen peroxide production independent of the phosphoinositide 3-kinase-Akt pathway. Cancer Res. 2005; 65(14):6264-6274.

24. Morehouse LA, Tien M, Bucher JR and Aust SD. Effect of hydrogen peroxide on the initiation of microsomal lipid peroxidation. Biochem Pharmacol. 1983; 32(1):123-127.

25. Bitew T, Sveen CE, Heyne B and Zaremberg V. Vitamin E prevents lipid raft modifications induced by an anti-cancer lysophospholipid and abolishes a Yap1-mediated stress response in yeast. J Biol Chem. 2010; 285(33):2573125742.

26. Visco C, Tzankov A, Xu-Monette ZY, Miranda RN, Tai YC, Li Y, Liu WM, d'Amore ES, Li Y, Montes-Moreno S, Dybkaer K, Chiu A, Orazi A, Zu Y, Bhagat G, Wang HY, et al. Patients with diffuse large B-cell lymphoma of germinal center origin with BCL2 translocations have poor outcome, irrespective of MYC status: a report from an International DLBCL rituximab-CHOP Consortium Program Study. Haematologica. 2013; 98(2):255-263.

27. Satterwhite E, Sonoki T, Willis TG, Harder L, Nowak R, Arriola EL, Liu H, Price HP, Gesk S, Steinemann D, Schlegelberger B, Oscier DG, Siebert R, Tucker PW and Dyer MJ. The BCL11 gene family: involvement of BCL11A in lymphoid malignancies. Blood. 2001; 98(12):3413-3420.

28. Czabotar PE, Lessene G, Strasser A and Adams JM. Control of apoptosis by the BCL-2 protein family: implications for physiology and therapy. Nat Rev Mol Cell Biol. 2014; 15(1):49-63.

29. D'Amelio M, Tino E and Cecconi F. The apoptosome: emerging insights and new potential targets for drug design. Pharm Res. 2008; 25(4):740-751.

30. Hausmann G, O'Reilly LA, van Driel R, Beaumont JG, Strasser A, Adams JM and Huang DC. Pro-apoptotic apoptosis protease-activating factor 1 (Apaf-1) has a cytoplasmic localization distinct from Bcl-2 or Bcl-x(L). J Cell Biol. 2000; 149(3):623-634.
31. Watanabe T, Hirota Y, Arakawa Y, Fujisawa H, Tachibana O, Hasegawa M, Yamashita J and Hayashi Y. Frequent LOH at chromosome 12q22-23 and Apaf-1 inactivation in glioblastoma. Brain Pathol. 2003; 13(4):431-439.

32. Huerta S, Heinzerling JH, Anguiano-Hernandez YM, Huerta-Yepez S, Lin J, Chen D, Bonavida B and Livingston EH. Modification of gene products involved in resistance to apoptosis in metastatic colon cancer cells: roles of Fas, Apaf-1, NFkappaB, IAPs, Smac/DIABLO, and AIF. J Surg Res. 2007; 142(1):184-194.

33. Gupta $\mathrm{N}$ and DeFranco AL. Lipid rafts and B cell signaling. Semin Cell Dev Biol. 2007; 18(5):616-626.

34. Brown KD, Hostager BS and Bishop GA. Differential signaling and tumor necrosis factor receptor-associated factor (TRAF) degradation mediated by CD40 and the Epstein-Barr virus oncoprotein latent membrane protein 1 (LMP1). J Exp Med. 2001; 193(8):943-954.

35. Longnecker $\mathrm{R}$ and Miller CL. Regulation of EpsteinBarr virus latency by latent membrane protein 2 . Trends Microbiol. 1996; 4(1):38-42.

36. Kawano R, Ohshima K, Karube K, Yamaguchi T, Kohno S, Suzumiya J, Kikuchi M and Tamura K. Prognostic significance of hepatocyte growth factor and c-MET expression in patients with diffuse large B-cell lymphoma. Br J Haematol. 2004; 127(3):305-307.

37. Higuchi M, Izumi KM and Kieff E. Epstein-Barr virus latent-infection membrane proteins are palmitoylated and raft-associated: protein 1 binds to the cytoskeleton through TNF receptor cytoplasmic factors. Proc Natl Acad Sci U S A. $2001 ; 98(8): 4675-4680$.

38. Smith TF, Gaitatzes C, Saxena K and Neer EJ. The WD repeat: a common architecture for diverse functions. Trends Biochem Sci. 1999; 24(5):181-185.

39. Kagan VE, Gleiss B, Tyurina YY, Tyurin VA, ElenstromMagnusson C, Liu SX, Serinkan FB, Arroyo A, Chandra $\mathrm{J}$, Orrenius $\mathrm{S}$ and Fadeel B. A role for oxidative stress in apoptosis: oxidation and externalization of phosphatidylserine is required for macrophage clearance of cells undergoing Fas-mediated apoptosis. J Immunol. 2002; 169(1):487-499.

40. Hirpara JL, Seyed MA, Loh KW, Dong H, Kini RM and Pervaiz S. Induction of mitochondrial permeability transition and cytochrome $\mathrm{C}$ release in the absence of caspase activation is insufficient for effective apoptosis in human leukemia cells. Blood. 2000; 95(5):1773-1780. 